(C) 2020, American Psychological Association. This paper is not the copy of record and may not exactly replicate the final, authoritative version of the article. Please do not copy or cite without authors' permission. The final article will be available, upon publication, via its DOI: $10.1037 /$ pag0000456 


\title{
The impact of age on the temporal compression of daily life events in episodic memory
}

\author{
Adrien Folville, Olivier Jeunehomme, Christine Bastin \& Arnaud D’Argembeau
}

University of Liège

Running head: Aging and temporal compression

Adrien Folville, GIGA-CRC In-Vivo Imaging, University of Liège, Liège, Belgium; Department of Psychology, Psychology and Neuroscience of Cognition Research Unit, University of Liège. Olivier Jeunehomme, Department of Psychology, Psychology and Neuroscience of Cognition Research Unit, University of Liège.

Christine Bastin, GIGA-CRC In-Vivo Imaging, University of Liège, Liège, Belgium; Department of Psychology, Psychology and Neuroscience of Cognition Research Unit, University of Liège.

Arnaud D'Argembeau, GIGA-CRC In-Vivo Imaging, University of Liège, Liège, Belgium; Department of Psychology, Psychology and Neuroscience of Cognition Research Unit, University of Liège.

Olivier Jeunehomme, Christine Bastin, and Arnaud D'Argembeau are, respectively, Postdoctoral Researcher, Research Associate, and Senior Research Associate at the F.R.S.-FNRS.

Corresponding author: Adrien Folville, GIGA-CRC In vivo Imaging, University of Liège, Allée du 6 Août, 8, B30, 4000 Liège, Belgium Phone: ++ 32 (0)4 36623 62, Fax: ++ 32 (0)4 36629 46, Email: adrien.folville@uliege.be This work was supported by the Fund for Research in Human Science and the National Fund for Scientific Research (FRESH/FRS-FNRS).

The authors wish to thanks Librairie Pax and Darius café in which parts of the Experiment took place. The data reported in this manuscript are available at https://osf.io/sdhqf/

Results of this study were presented at the meeting of the Belgian Association for Psychological Science, $14^{\text {th }}$ of May 2019, Liège Belgium as well as at the conference: The dynamic and flexible nature of memories, on the $7^{\text {th }}$ and $8^{\text {th }}$ of June 2019, in Lyon, France. 


\begin{abstract}
While age differences in episodic memory are well documented, the impact of age on the structure of memories for real-world events has not been investigated in detail. Recent research has shown that the continuous flow of information that constitutes daily life events is compressed in episodic memory, such that the time needed to mentally replay an event is shorter than the actual event duration. To examine whether this process of temporal compression of prior experience in episodic memory is affected by aging, we asked young and older adults to engage in a series of events that simulated daily life activities while their experience was automatically recorded using a wearable camera. Subsequently, participants were asked to mentally replay these events in as much detail as possible and then to verbally report recalled contents and to rate the subjective qualities of their memories. Results revealed that the rates of temporal compression of events during mental replay were similar in young and older adults. In both age groups, rates of temporal compression were predicted by the density of recalled moments of prior experience per unit of time of the actual event duration. Interestingly, however, the number of recalled moments predicted the subjective vividness of memories in young but not in older adults. Taken together, these results suggest that the process of temporal compression of events in episodic memory is unaffected by age but that the subjective experience of memory vividness becomes less tied to recalled moments that represent the unfolding of events.
\end{abstract}

Key-words: Episodic memory; Aging; Temporal compression; Goals; Vividness Number of words: 7838 
Aging is associated with diminished episodic memory performance. Compared to young adults, older adults remember past events in less detail and have particular difficulty in reinstating the context of prior experiences (Old \& Naveh-Benjamin, 2008; Spencer \& Raz, 1995). Relatively little is known, however, about age effects on the temporal structure of memories for real-life events-how the continuous flow of experience is summarized in long-term memory. The main reason for this gap in knowledge is that previous studies on age-related differences in episodic memory have mainly used discrete laboratory stimuli (such as pictures or words) that are devoid of temporal dynamics. Recent research in young adults suggest that episodic memories represent prior experience in a temporally compressed form, such that remembering an event takes less time than the actual event duration (Bonasia, Blommesteyn, \& Moscovitch, 2016; Jeunehomme \& D’Argembeau, 2019; Michelmann, Staresina, Bowman, \& HansImayr, 2019). The aim of the current study was to examine whether this phenomenon of temporal compression of events in episodic memory is affected in normal aging.

\section{Temporal compression of events in episodic memory}

Our daily life is made of a continuous flow of events and experiences. Episodic memory retains traces of these events, allowing us to mentally re-experience the past (Tulving, 2002). However, memories are not literal records of past episodes but instead summary representations of prior experience (Conway, 2009). Here, we focus on how the unfolding of events is summarized in memory by investigating how event memories map onto the real time it took for events to unfold (Jeunehomme \& D'Argembeau, 2019; Wang \& Gennari, 2019). We use the term "temporal compression" to refer to the fact that the representation of the unfolding of events in memory often does not map onto the original event duration: 
the time it takes to remember an event is typically shorter than the actual duration of this event in the past.

Recent studies have capitalized on wearable camera technology to examine this compression phenomenon when remembering real-world events (Jeunehomme \& D’Argembeau, in press, 2019; Jeunehomme, Folville, Stawarczyk, Van der Linden, \& D’Argembeau, 2018). In one study, young adults engaged in a series of daily-life activities (e.g., buying a newspaper) and were later asked to mentally replay each event in as much detail as possible (Jeunehomme \& D'Argembeau, 2019). It was found that remembering an event took less time than the actual event duration, showing that the unfolding of past experience is temporally compressed in episodic memory (Arnold, laria, \& Ekstrom, 2016; Baldassano et al., 2017; Bonasia et al., 2016; Michelmann et al., 2019). Furthermore, verbal reports on the content of memories suggested that this compression occurs, at least in part, because events were represented as a succession of short-time slices of prior experience that were separated by temporal gaps (i.e., some moments of prior experience were not remembered; Jeunehomme et al., 2018; see also Michelmann et al., 2019). The density of remembered moments of experience per unit of time of the actual event duration predicted rates of temporal compression during mental replay. Importantly, however, rates of temporal compression were not constant but varied according to the nature of remembered events. Specifically, events involving actions (e.g., buying a drink at the cafeteria) were less compressed than events involving spatial displacements (e.g., going from one place to another with no particular action to perform other than walking) (Jeunehomme \& D’Argembeau, 2019; Jeunehomme et al., 2018). 
Together, these studies show that episodic memories are not replicas of prior experiences but represent the unfolding of events in a form that does not coincide with the actual event duration. While the mechanisms underlying this temporal compression of events in episodic memory remain to be investigated in detail, at least two factors may determine compression rates. First, compression rates may depend on how events are segmented and structured at encoding. According to event segmentation theory, the continuous stream of experience is perceived by means of event models that represent the ongoing situation (Kurby \& Zacks, 2008; Zacks, Speer, Swallow, Braver, \& Reynolds, 2007). Event models are constantly updated following perceptual or conceptual changes in experience (e.g., changes in location, character, object, goals, actions, and so forth), resulting in the formation of event boundaries - the perception that an event has ended and another event begins - that delimit distinct memory traces (Clewett \& Davachi, 2017; Radvansky \& Zacks, 2017; Zacks et al., 2007). Thus, compression rates when representing the unfolding of events may depend on the density of event segments per unit of time: the more segments are perceived at encoding, the less events are compressed in memory (Faber \& Gennari, 2015; Jeunehomme \& D'Argembeau, in press). Second, rates of temporal compression may be flexibly modulated at retrieval according to task demands and contextual factors (Wang \& Gennari, 2019). For example, people may modulate the granularity of event representations depending on whether they need to remember the gist or details of previous action sequences. These two factors are not mutually exclusive but their respective contributions to the temporal compression of events is still unclear. 


\section{Age-related changes in episodic memory}

The impact of age on episodic memory is well documented, with many studies showing that the ability to remember events declines with increasing age (for reviews, see Cabeza, Nyberg, \& Park, 2016; Koen \& Yonelinas, 2014). This decline has been attributed to a diminished capacity to retrieve associations between the different features constituting events (Chalfonte \& Johnson, 1996). Notably, laboratory studies have shown that older adults have objectively less accurate episodic memories, as indexed by source memory (Bastin et al., 2014; Duarte, Henson, \& Graham, 2008; Duarte, Ranganath, Trujillo, \& Knight, 2006) or free-recall (Norman \& Schacter, 1997) tasks. Similarly, studies on memory for reallife events have shown that older adults have impoverished memory representations relative to young adults (Hashtroudi, Johnson, \& Chrosniak, 1990; Madore, Gaesser, \& Schacter, 2014; Robin \& Moscovitch, 2017; St-Jacques, Rubin, \& Cabeza, 2012). Interestingly, however, recent studies did not find any age difference in the richness of recent memories (between two weeks and one year old) for real-life events (Aizpurua \& Koutstaal, 2015; Mair, Poirier, \& Conway, 2017).

It is also worth noting that in some studies, the reduced level of detail observed in older adults' verbal reports of their memories contrasted with their subjective memory ratings, which were either similar or even higher than the ratings of young adults (Folville et al., 2019; Folville, D’Argembeau, \& Bastin, in press; Hashtroudi et al., 1990; Robin \& Moscovitch, 2017; St-Jacques et al., 2012; St-Laurent, Abdi, Bondad, \& Buchsbaum, 2014).

Although these studies provide important insights into the impact of aging on the richness of episodic memories, they did not investigate the temporal structure of memories. To the best of our knowledge, no study has investigated whether young and older adults 
compress the continuous flow of experience in the same manner in episodic memory representations.

\section{Aging and event cognition}

When segmenting videos depicting daily life activities, older adults have lower segmentation agreement (i.e., the participant's segmentation maps onto the segmentation pattern of the whole sample to a reduced extent) and the segments they identify are less hierarchically organized (i.e., fine-grained segmentation maps less well onto coarse-grained segmentation) relative to young adults (Kurby \& Zacks, 2011; Zacks, Speer, Vettel, \& Jacoby, 2006). These results suggest that the stream of real-life events may be less well-structured and less likely to be segmented into distinct units among older adults, which could in turn impact the temporal compression of events in episodic memory.

However, other studies indicate that older adults do not necessarily have lower segmentation abilities. For instance, studies of reading comprehension have shown that reading speed decreases at event boundaries - because cognitive resources are allocated to event model updating -in a similar way in young and older adults (Radvansky, Zwaan, Curiel, \& Copeland, 2001), and a recent study has shown that the neural correlates of event segmentation do not differ between young and older adults (Kurby \& Zacks, 2018b). Furthermore, older adults can display similar rates of event segmentation as young adults, for instance when they segment narrative texts or picture stories (Magliano, Kopp, Mcnerney, Radvansky, \& Zacks, 2012). The authors proposed that older adults rely on their knowledge of events to adequately process relevant event features. As a matter of fact, situation models (i.e., the understanding of the characters, physical environment, and spatio-temporal framework of a narrative or a situation) are not affected by healthy aging 
(Radvansky \& Dijkstra, 2007). For example, in many studies, young and older adults did not differ in their comprehension and memory for situation narratives (Radvansky \& Curiel, 1998; Radvansky et al., 2001; Stine-Morrow, Soederberg Miller, \& Leno, 2001). Together, these findings suggest that older adults have preserved event cognition and that situation models may help them segmenting the continuous stream of experience in a similar way as young adults do. Therefore, it could be that, despite a general reduction in level of detail, older adults' episodic memories display a temporal structure that is comparable to young adults' memories, notably in terms of rates of event compression.

\section{The current study}

The aim of the current study was to examine the impact of age on the temporal compression of daily-life events in episodic memory. To do so, we used an experimental paradigm that capitalizes on wearable camera technology (Jeunehomme \& D’Argembeau, in press; 2019). Young and older adults engaged in a series of events that simulated daily-life activities while wearing a camera that automatically recorded their experience from the first person perspective (Chow \& Rissman, 2017). They then had to mentally replay each event in as much detail as possible and the temporal compression of the event in memory was estimated as the ratio of the actual event duration to the duration of mental replay. Following mental replay, participants were asked to verbally describe everything they remembered during their mental replay, which allowed us to assess the density of recalled segments of prior experience per unit of time of the actual event duration. We predicted that the density of recalled moments of experience would predict temporal compression rates (Jeunehomme \& D'Argembeau, 2019) and examined whether this was the case in both age groups. 
Another goal of this study was to investigate whether variations in temporal compression rates for different types of events are similar in young and older adults. In young adults, rates of temporal compression have been found to be lower for events that involve specific actions than for events that involve spatial displacements (Jeunehomme \& D’Argembeau, 2019; Jeunehomme et al., 2018). This was explained by the fact that memories for actions contained a higher density of moments of experience per unit of time than spatial displacements, so that the mental replay of the former events took proportionally more time (and thus was less compressed) than the mental replay of the latter events. Here we assessed whether this pattern of results would also be observed in older adults.

The third aim of the current study was to examine the impact of age on the subjective experience of remembering and its relation to the temporal structure of memories. Jeunehomme and D'Argembeau (2019) found that the subjective sense of reexperiencing an event during mental replay was predicted by the number of retrieved moments of prior experience. Previous studies have shown that older adults provide similar or even higher subjective vividness ratings than young adults when remembering real-life events (Comblain, D’Argembeau, \& Van Der Linden, 2005; St-laurent, Abdi, Burianova, \& Grady, 2011), even when they have a lower memory for the events (Gallo, Korthauer, Mcdonough, Teshale, \& Johnson, Elizabeth, 2011; Robin \& Moscovitch, 2017; St-Jacques et al., 2012) and less specific neural representations (Folville et al., 2019). Besides, a recent study showed that older adults' subjective vividness ratings are less tied to the amount of retrieved episodic details (Folville et al., in press). Consequently, we hypothesized that the subjective experience of remembering would be less tied to the objective number of recalled moments of prior experience in older than young adults. 


\section{Method}

\section{Participants}

Thirty-four young (mean age $=22.85$ years, $S D=2.96$, range $19-29$ years; 13 males) and 34 older (mean age $=68.32$ years, $\mathrm{SD}=4.19$, range $61-76$ years; 22 males) adults took part in the study. This sample size was determined a priori using G*Power 3 (Faul, Erdfelder, Lang, \& Buchner, 2007) to achieve a statistical power of $80 \%$ to detect a significant within-group difference in compression rates (comparing actions and spatial displacements), considering an alpha of .05 and a medium effect size (Cohen's $d=0.50)$. Furthermore, this sample size provided a statistical power above $95 \%$ to detect age differences, considering an alpha of .05 and the effect size reported by Madore, Gaesser and Schacter (2014) for the recall of episodic details from real-life events (Cohen's $d=1.46$ ). Exclusion criteria were past/current neurological or psychiatric disorders and any medication that could interfere with cognitive functioning. Two participants were excluded and replaced by two other participants: one young adult because he guessed that his memory would be tested and one older adult because he voluntary terminated the testing session before the end. Groups did not differ in terms of education, $t(66)=-1.26, p=.211$ ( $\mathrm{M}_{\text {young }}=14.15, \mathrm{SD}_{\text {young }}=1.94 ; \mathrm{M}_{\text {older }}=15.09$, $\mathrm{SD}_{\text {older }}=3.88$ ). Older adults' general cognitive functioning was assessed with the Dementia Rating Scale (DRS; Mattis, 1976) and all older participants performed within the agecorrected norms of the DRS: $M=139.59, S D=3.39$ (Pedraza et al., 2010). On the Mill-Hill vocabulary questionnaire (Deltour, 1993), scores indicated better performance in older than in young adults, $t(66)=-6.51, p<.001$ ( $\left.\mathrm{M}_{\text {young }}=24.09, \mathrm{SD}_{\text {young }}=4.49 ; \mathrm{M}_{\text {older }}=29.76, \mathrm{SD}_{\text {older }}=2.37\right)$. All participants gave written informed consent and the study was approved by the Ethics Committee of the Faculty of Psychology of the University of Liège, Belgium. 


\section{Materials and Procedure}

The experimental procedure consisted of two phases: participants first experienced a series of events that simulated daily life activities and then received a memory task in which they had to mentally replay these events, to describe remembered information and to rate their subjective experience while remembering.

Experienced events. Participants were instructed to perform different actions in a predefined order in various locations around the main building of the university in the city centre of Liège (Belgium), while the content and timing of their experience was recorded using a wearable camera: the Autographer (OMG Life Ltd.). Participants were not aware that their memory would be subsequently tested and it was explained that we were pre-testing a new wearable camera for use in future studies aiming at investigating people's experience in their daily life. Participants received written instructions describing the walk, which they had to remember. Before starting the walk, they were asked to recite all the steps to ensure that they remembered and understood all actions and spatial displacements. During the entire walk, participants wore the Autographer tied around their neck and the camera automatically took pictures from a first-person perspective according to several parameters (e.g. luminosity, body movements). In the current study, the camera was set to capture the pictures at the maximum rate (around ten pictures per minute).

The walk was composed of two types of events: actions in which participants interacted with people and objects (circles on Figure 1) and spatial displacements in which participants walked from one place to another (lines on Figure 1). The order of the events was the same for all participants and was as follows (see Figure 1): participants first left the 
testing room and went downstairs to the main hall of the building (note that in the subsequent memory task this part of the walk was used as a practice trial and was not included in the analyses). When they arrived in the hall, participants were instructed to choose a leaflet on the display stands. The leaflets depicted activities that could be done in Liège and its area (e.g. museum visits or exhibitions) and participants were instructed to choose the activity they would like to carry out in a near future. Next, participants left the University building and went to a bookshop to buy a card (e.g., a postcard or greetings card). After having bought the card, participants were asked to go to a cafe to purchase the beverage of their choice that they were instructed to take away. Next, they went back to the University building and went to the reception office to ask the closing time of the building. Finally, they went back to the testing room. Before the walk, participants received 5 euros to purchase the card and the beverage.

\section{< Figure 1 about here >}

Memory task. Immediately after the walk, the real aim of the study was revealed to the participants and they were asked whether they had guessed that their memory would be tested. While participants filled in the Mill-Hill vocabulary test and read the memory task instructions, the experimenter uploaded the pictures that had been taken by the camera on the computer. The task was programmed using E-Prime 2.0 software (Psychology Software Tools, Pittsburgh, PA). The task comprised eight trials (four actions and four spatial displacements; see Figure 1) that were presented in a random order. Each trial began with the presentation of two pictures and a label describing one segment of the walk (e.g., going to the bookshop; see Figure 2). The left and right pictures corresponded respectively to the beginning and end of an experienced event; the two pictures were separated by a black arrow oriented to the right, indicating their chronological order. Once participants identified 
the corresponding event, they were instructed to close their eyes and to mentally relive the event as precisely as they could (i.e., everything that happened from the moment represented by the picture on the left to the moment represented by the picture on the right). Participants were instructed to press the space bar to indicate that they started their mental replay and as soon as they had mentally re-experienced the event, they pressed the space bar again to indicate the end of their mental replay. Next, they were presented with six rating scales assessing the vividness of their memory, the sense of reliving the event, the amount of visual details, spatial information, and thoughts, and memory for the order of the event. Each Likert scale ranged from 0 (not vivid/vague) to 5 (very vivid/very clear) and scale order was randomised for each trial. No time limit was imposed for making these ratings. After the last rating scale, participants were asked to verbally describe in as much detail as possible everything they remembered during their mental replay. Verbal reports were recorded. Once participants finished their recall, they pressed the space bar to move to the next trial.

Before starting the task, all participants performed a practice trial with the first part of the walk (i.e. leaving the testing room and going downstairs) to familiarise them with the procedure and the response buttons. During this practice trial, care was taken to ensure that participants had correctly understood each part of the memory task.

After the memory task, participants received a video description task that was used as a measure of their narrative style. They were presented with two videos and were instructed to verbally describe each video while it was played on the computer screen. Each video was filmed from a first-person perspective and participants were asked to describe what was happening in the video as if they were experiencing the events. Their descriptions were recorded. Videos represented events that happened in a different environment than 
the main experiment to avoid possible interference from previously experienced events. One video displayed someone doing shopping and the other video displayed someone walking in the street. The order of video presentation was randomised. The video descriptions were used to measure participants' narrative style as it has been shown that older adults tend to verbally report fewer details than young adults even when the task does not involve any episodic memory component (Gaesser, Sacchetti, Addis, \& Schacter, 2011).

Finally, participants filled in a questionnaire that assessed their familiarity with the different locations they visited during the main experiment. Each segment of the walk was rated using a Likert scale ranging from 1 (not familiar) to 7 (very familiar).

\footnotetext{
$<$ Figure 2 about here >
}

\section{Narrative coding}

Verbal descriptions of memories and videos were transcribed and were scored using the same procedure as previous studies investigating the temporal structure of episodic memories (Jeunehomme et al., 2018; Jeunehomme \& D'Argembeau, 2019). The verbal descriptions of remembered events consisted of a succession of moments or slices of prior experience (referred to as experience units) that typically represented the unfolding of events in a chronological order. The identification of an experience unit was based on transitions (e.g., linking words such as then or next) and breaks (moments of silence) in the discourse of the participant. For instance, a typical recall protocol involved the following succession of experience units: "I left the bookshop and turned right" (first experience unit), "while walking, I saw a woman with an umbrella" (second experience unit), "then, I crossed 
the pedestrian path" (third experience unit), "next, I walked along the organic shop" (fourth experience unit), and so forth. Each of these experience units included one or several pieces of information (referred to as unit components), which described various aspects of experience that could involve the external environment, mental states, and actions. The content of each experience unit was coded by categorizing unit components in eight categories: person, object, thought, action with interaction, spatial movement, perceptual detail, spatial detail, and comment (each category is described in detail in Table 1, along with examples). These categories were mutually exclusive (i.e., a given component was classified in only one category), but an experience unit could include multiple components (e.g., a person, an object, and a perceptual detail). For instance, the experience unit "while walking, I saw a woman with an umbrella" contains a spatial displacement component (“while walking"), a person component ("a woman"), and an object component ("with an umbrella"). Thanks to the pictures taken during the walk, we could verify the accuracy of the majority of the reported episodic details to ensure that participants' recall did not contain any intrusion. However, some components could not be verified because they involved elements outside the field of view of the camera.

$<$ Table 1 about here >

The scoring of experience units and components was performed by the first author and a second trained rater independently scored a randomly selected $20 \%$ of the data of each group to assess scoring reliability. The identification of experience units showed an excellent agreement between the two raters: ICC $=.98($ Koo \& Li, 2016). For unit components, there was excellent agreement regarding persons, ICC $=.90$, and thoughts, ICC $=.93$, and good agreement regarding objects, ICC $=.79$, actions with interaction, ICC $=.75$, 
spatial movements, ICC $=.77$, perceptual details, ICC $=.81$, spatial details, ICC $=.82$ and comments, ICC $=.86$.

Similar coding procedures for both experience units and unit components were applied to video descriptions, with the exception that the thought category was not used (no thought was described). Again, all the data were scored by the first author and the second trained rater independently scored $20 \%$ of the data of each group. ICCs were excellent regarding experience units, ICC $=.92$, persons, ICC $=.91$, objects, ICC $=.92$, spatial movements, ICC $=.93$, perceptual details, ICC $=.93$, spatial details, ICC $=.91$ and comments, ICC $=.91$, and were good regarding actions with interaction, ICC $=.87$ (Koo \& Li, 2016).

\section{Statistical analyses}

The rate of temporal compression of an event during mental replay was measured as the ratio of the actual duration of the event (measured as the time separating the shooting moments of the two pictures depicting the beginning and end of the event) to the duration of its mental replay. Because the distribution of this measure of temporal compression was substantially skewed, we used robust statistical methods to analyse the data. Robust statistical methods perform well in terms of type I error control and statistical power, even when the normality and homoscedasticity assumptions are violated, and thus they increase the likelihood of discovering genuine differences between groups and associations among variables (Wilcox, 2012). More specifically, we conducted robust $2 \times 2$ mixed analyses of variances (ANOVAs) to investigate the effects of age and type of events (i.e., actions vs. spatial displacements) on temporal compression rates, recalled experience units, and recalled components within units. These robust ANOVAs were conducted using the $20 \%$ 
trimmed means (a robust measure of location that ignores the top and bottom $20 \%$ of data) and 2000 bootstrap samples (as a way to deal with bias in standard errors by estimating the shape of the sampling distribution by sampling with replacement from the data), as recommended by Field and Wilcox (2017). Effect sizes were estimated using the explanatory measure of effect size $\xi$; values of $0.10,0.30$, and 0.50 correspond to small, medium, and large effect sizes (Mair \& Wilcox, 2019). These analyses were performed using the functions of Wilcox (2012) implemented in R (R Core Team, 2013).

To investigate whether temporal compression rates were predicted by the density of recalled experience units, we conducted a robust multilevel regression analysis (two-level random intercept model with events as level 1 units and participants as level 2 units) using the robustlmm package in $\mathrm{R}$ (Koller, 2016). Compression rate was the outcome variable and age group, density of recalled experience units, and their interaction were predictors. Similar robust multilevel regression analyses were used to investigate the effects of recalled information and age on the subjective experience of remembering.

The alpha level was set at .05 in all analyses. All descriptive statistics refer to the $20 \%$ trimmed means and their $95 \%$ confidence intervals ( $\mathrm{Cls})$ calculated using the percentile bootstrap method with 2000 bootstrap samples (Wilcox, 2012).

\section{Results}

\section{Temporal compression of events during mental replay}

The mean temporal compression of events involving actions and spatial displacements are shown in Figure 3 for the two age groups. A robust 2 (age) $\times 2$ (event type) mixed ANOVA revealed a main effect of the type of events on compression rates, $F_{t}(1,41.65)=8.47, p=$ 
$.006, \xi=0.17$, showing that actions were less compressed in memory than spatial displacements. The main effect of age and the age by event type interaction were not significant, $F_{t} s<1^{1}$. These results could not be simply explained by the influence of event duration on compression rates (see Supplementary material).

$<$ Figure 3 about here >

\section{Density and components of recalled experience units}

Verbal descriptions of remembered events consisted of a succession of moments of prior experience (experience units). To assess the density of recalled experience units during mental replay, we computed the number of reported experience units per unit of time of the actual event duration. The mean density of experience units (i.e., the number of recalled units per minute of the actual event duration) for actions and spatial displacements are shown in Figure 4 for the two age groups. A robust 2 (age) $\times 2$ (event type) mixed ANOVA revealed a main effect of the type of events, $F_{t}(1,41.96)=99.22, p<.001, \xi=0.79$, showing that participants recalled a higher density of experience units for actions than spatial displacements. Young adults recalled slightly more experience units than older adults, but the effect was not statistically significant, $F_{t}(1,41.93)=3.31, p=.076, \xi=0.30$. The age by type of event interaction was not significant, $F_{t}<1$.

< Figure 4 about here >

To investigate the impact of age and type of events on the number of components within experience units, a robust 2 (age) $\times 2$ (event type) mixed ANOVA on the total number

\footnotetext{
${ }^{1}$ Note that similar results were obtained when analysing the ratio of memory duration to actual event duration.
} 
of components reported per experience unit was conducted (all types of components were summed for this analysis, except comments). It revealed a main effect of event type, $F_{t}(1$, 40.01) $=33.15, p<.001, \xi=0.55$, showing that actions contained more components than spatial displacements. Neither the main effect of age, $F_{t}(1,37.29)=0.01, p=.98, \xi=0.02$, nor the age $x$ event type interaction, $F_{t}(1,37.29)=0.05, p=.81$, were significant. Separate analyses conducted on each type of component can be found in the Supplementary material.

\section{Relationship between temporal compression rates and recalled experience units}

The preceding analyses indicated that the rates of temporal compression of events in episodic memory and the density of recalled experience units were similar in young and older adults. Next, we sought to replicate previous findings that temporal compression rates were predicted by the density of recalled experience units (Jeunehomme \& D'Argembeau, 2019) and further examined whether this relationship was similar in young and older adults. We conducted a robust multilevel regression analysis with compression rate as dependent variable and with the density of recalled experience units and age group as first- and second-level predictors; the density of experience units by age group cross-level interaction was also entered as predictor to investigate potential age differences in the relationship between temporal compression rates and the density of recalled experience units. This analysis revealed that the density of recalled experience units significantly predicted temporal compression rates, $\beta=-0.162, S E=0.019, t=-8.14, p<.001$, indicating that memories that included a higher density of experience units were less compressed. The effects of age, $\beta=0.003, S E=0.069, t=0.06$, and the density of experience units by age group interaction, $\beta=-0.015, S E=0.028, t=-0.56$, were not significant. 


\section{Subjective experience of remembering}

Another goal of this study was to examine the impact of age on subjective memory judgements and to determine whether these judgements were predicted by recalled experience units. We conducted a robust multilevel regression analysis with vividness ratings as outcome variable and with the number of recalled experience units and age group as first- and second-level predictors; the number of recalled units by age group cross-level interaction was also entered as predictor in the model to examine potential age differences in the relationship between subjective vividness and the number of retrieved experience units. Finally, the total number of components recalled within experience units was also entered in the model to examine its potential contribution to subjective vividness. We found that the subjective vividness of memories was significantly predicted by the number of recalled experience units and by age, with older adults providing higher vividness judgements than young adults (see Table 2). The cross-level interaction was also significant and follow-up analyses ran on each age-group separately revealed that the number of recalled experience units predicted vividness ratings in young adults, $\beta=0.164, S E=0.061, t$ $=3.13, p<.01$, but not in older adults, $\beta=0.022, S E=0.058, t=0.37$. The number of components within experience units did not significantly predict vividness ratings (Table 2 ).

A similar analysis was conducted with the subjective sense of reliving as outcome variable. The effects of the number of experience units and age group were significant (see Table 2). However, the cross-level interaction was not significant, meaning that the number of recalled experience units predicted the subjective experience of reliving to a similar extent in young and older adults. Again, the number of components within experience units 
did not significantly predict subjective memory. Similar results were found for ratings of visual details, spatial information, and thoughts (see Supplementary material).

$<$ Table 2 about here $>$

\section{Video descriptions and familiarity with the walk environment}

Analyses concerning the descriptions of the videos revealed that the density of experience units (i.e., the number of experience units described per minute of video) did not differ between age groups, $\mathrm{M}_{\text {diff }}=0.668,95 \% \mathrm{Cl}[-0.84,2.18], Y t(33.45)=0.85, p=.37^{2}, \xi=0.18$ (20\% trimmed means: $M_{\text {older}}=9.55 ; M_{\text {young }}=10.22$ ). Familiarity ratings with the walk environment differed between young and older adults for the "buying the beverage" event $(p=.006)$, while no age-difference was found for the remaining events (all $p \mathrm{~s}>.13$ ). To ensure that differences in the familiarity of the "buying the beverage" event did not influence the results, all the analyses presented above were re-conducted while controlling for this difference. The pattern of results remained unchanged.

\section{Discussion}

This study is the first to examine the impact of age on the temporal structure of memories for complex, real-world events. Young and older adults engaged in a series of events that simulated daily life activities while their experience was automatically recorded using a wearable camera. They were then asked to mentally replay each event in as much detail as possible, to evaluate their subjective experience of remembering, and to verbally describe the content of recalled information. We found that the rate of temporal compression of

\footnotetext{
${ }^{2}$ Video descriptions of one older participant were unavailable due to recording malfunction.
} 
events during mental replay did not differ between age groups but was lower for actions than spatial displacements. The density and components of recalled experience units also did not significantly differ between age groups. Critically, the density of recalled experience units predicted temporal compression rates to the same extent in both age groups. However, subjective ratings of memory vividness were higher in older than young adults, and the number of recalled experience units predicted vividness ratings in young but not in older adults.

\section{The temporal compression of events in episodic memory}

The main finding of the current study is that the rate of temporal compression of events in episodic memory (i.e., how fast events are mentally replayed relative to the actual event duration) did not differ significantly between age groups. Remembering an event typically takes less time than the initial experience, suggesting that the unfolding of prior experience is compacted in memory (Baldassano et al., 2017; Chen et al., 2017; Jeunehomme \& d'Argembeau, 2019; Michelmann et al., 2019). While the exact nature of this compression mechanism remains to be investigated, previous studies suggest that memory compression occurs, at least in part, because of temporal discontinuities in the mental representation of the unfolding of events (Jeunehomme et al., 2018; Jeunehomme \& D'Argembeau, 2019). Specifically, event memories are not replicas of the continuous stream of prior experience but represent the dynamic unfolding of events as a succession of discrete moments of experience (here referred to as experience units) that are separated by temporal gaps (i.e., some moments of prior experience are not remembered, such that people mentally jump from one moment to another without representing what happened in between). The magnitude of temporal compression may depend on the length of these temporal gaps 
during mental replay or, reciprocally, on the density of remembered experience units per unit of time of the actual event duration (Jeunehomme \& D'Argembeau, 2019). In line with this view, the present results replicated previous findings that rates of temporal compression were predicted by the density of recalled experience units (Jeunehomme \& D'Argembeau, 2019) and further showed this was the case in both age groups, suggesting that older adults compress the continuous flow of their experience in episodic memory in the same way as young adults do.

Event segmentation processes play a key role in the formation of the experience units that constitute episodic memories. According to event segmentation theory (Kurby \& Zacks, 2008; Zacks et al., 2007), the segmentation of the continuous stream of experience into distinct events follows perceptual or conceptual changes in ongoing experience, which are interpreted as event boundaries. Thus, the rate of event segmentation is modulated by the characteristics and structure of events (Kurby \& Zacks, 2011), which in turn determine the rate of compression during subsequent episodic remembering (Faber \& Gennari, 2015; Jeunehomme \& D’Argembeau, in press). Some previous studies have found that older adults have lower event segmentation agreement than young adults, and that the event segments they identify are less hierarchically organized (Kurby \& Zacks, 2011; Magliano et al., 2012; Zacks et al., 2006). At the same time, aging does not necessarily lead to lower segmentation rates (Magliano et al., 2012) and the processing of situation models is largely preserved with increasing age (Radvansky \& Dijkstra, 2007). Knowledge about situation models may thus compensate for older adults' reduced capacity to detect perceptual changes in the stream of conscious experience, thereby helping them to identify event boundaries as efficiently as young adults (Magliano et al., 2012). In a recent study, both young and older adults were found to have lower memory for the object they were currently carrying when they walked 
through a door than when they moved within the same room (Radvansky, Pettijohn, \& Kim, 2015). Because moving from a room to another requires the updating of event models, which in turn causes memory interference (Radvansky, Krawietz, \& Tamplin, 2011), the authors concluded that the finding that young and older adults were equally sensitive to door passing is congruent with the idea that event cognition is preserved with increasing age (Radvansky et al., 2015).

Together, these studies suggest that older adults segment the continuous stream of their experience into distinct and meaningful experience units just as young adults do. These preserved segmentation abilities may thus explain the present results that the temporal structure and compression of episodic memories was similar in young and older adults. This interpretation is tentative, however, and further studies directly linking event segmentation and rates of temporal compression are needed to investigate the exact contribution of event segmentation processes to episodic memory compression in older adults.

While on average the unfolding of events is mentally replayed at a faster rate than the actual event duration, the experience units that compose memories (i.e., the slices of prior experience that are remembered) may in fact be replayed at the same speed as the original experience (see Michelmann et al., 2019), although they may sometimes be replayed faster or slower. Thus, the speed of mental replay of experience units within memories and its possible modulation remain to be investigated in detail. Besides, it is likely that rates of event compression depend on retrieval conditions. In particular, it would be interesting to investigate the extent to which the speed of mental replay can be controlled and adaptively modulated according to task demands and the context in which 
remembering takes place. For instance, events may be mentally replayed more or less quickly depending on whether one needs to remember minute details or the general structure of prior experience. Rates of temporal compression may thus be influenced by task instructions: in the current study, participants were asked to mentally relive events as precisely as they could, but asking participants to remember the general structure of events or asking them to recreate the original timing of experience might lead to different compression rates. ${ }^{3}$ Temporal compression rates may also be sensitive to context and statedependent effects, as well as event dimensions such as their emotionality and personal significance. The extent to which these variables influence young and older adults' episodic memory compression to a similar extent should be investigated in future studies.

\section{Richness of experience units within memories}

When examining the level of detail of recalled experience units, we found no age difference in the number of components constituting experience units, which suggests that the moments of experience remembered by older adults were as detailed as those recalled by young adults. This results is somewhat surprising given the plethora of previous studies (using either laboratory stimuli or real-life events) showing lower context memory or lower episodic recall in aging (Duarte et al., 2008; Gallo et al., 2011; Levine, Svoboda, Hay, Winocur, \& Moscovitch, 2002; St-Jacques et al., 2012; St-laurent et al., 2011). However, some methodological factors are important to take into account when considering our results.

\footnotetext{
${ }^{3}$ Interestingly, however, it seems that events are temporally compressed even when people are asked to recreate their original timing. Indeed, a recent study of Wang and Gennari (2019) showed that participants mentally replayed the unfolding of visual animations more quickly than their actual clock duration (at least for animations that lasted longer than 5-6 seconds), although they were asked to mentally replay the animations exactly as they occurred in their original time course.
} 
First, we used a very short delay between encoding and retrieval (i.e., 10 minutes), whereas most studies examining age differences in memory for real-world events used much longer delays. Interestingly, Mair et al. (2017) recently found that young and older adults did not differ in the amount and richness of episodic details when remembering recent (two-weeks old) real-life events. It could be that age differences in our paradigm would appear if longer retention intervals were used (see Jeunehomme et al., 2018, for data on memory for real-life events after different delays in young adults).

Second, it could be that the picture cues provided for each event helped older participants to reinstate detailed memory representations, notably because these retrieval cues may place less demand on older adults' declining executive function capacities for retrieving information from episodic memory (see Allé et al., 2017; Silva, Pinho, Macedo, \& Moulin, 2018, for evidence that pictures taken with life logging camera provide particularly powerful cues to aid episodic memory retrieval). Thus, it remains possible that age-related differences in episodic details would appear if more abstract (verbal) cues were used to elicit memories.

Third, it deserves mention that there is a large heterogeneity in cognitive aging (Cabeza, Anderson, Locantore, \& Mclntosh, 2002), and older adults with higher executive function capacities can perform as well as young adults in recollection tasks (Davidson \& Glisky, 2002). Thus, it could be that by chance or due to an unintentional sampling bias, the older adults participating in the present study were mainly high functioning individuals. The current study did not include a detailed neuropsychological assessment of participants' executive functions and replicating the current findings while controlling for individual differences in cognitive functioning would strengthen our conclusions. 


\section{Influence of types of events on memory compression rates}

Of interest is the finding that actions were associated with lower temporal compression rates (and a higher density of experience units) in episodic memory than spatial displacements, which replicates previous findings (Jeunehomme \& D'Argembeau, in press, 2019; Jeunehomme et al., 2018). Relative to spatial displacements, actions are more likely to be segmented in fine-grained subevents (Hard, Recchia, \& Tversky, 2011), leading to the formation of more experience units in episodic memory (Jeunehomme \& D'Argembeau, in press). Thus, the mental replay of actions may take more time than the mental replay of spatial displacements because there are more moments of experience to reinstate in the former than in the latter. Our finding that the difference in memory compression between these two kinds of events was similar in young and older adults provides further support to the view that the temporal structure of episodic memories is largely preserved in aging.

These results can also be interpreted from a functional perspective. According to Conway (2009), one of the main functions of episodic memories is to represent knowledge about specific actions and action outcomes, which provides a mean to check on recent progress with current goals and plans. Therefore, events may be retained in episodic memory as a function of the goal structure of an experience, such that memories would be less compressed when they involve goal-directed actions. On this view, the present results may indicate that the role of short-term goals in the selection of aspects of experience that are maintained in episodic memory is preserved in normal aging. Yet, recent evidence suggests that aging decreases the ability to perceive the goal structure of events (Kurby \& Zacks, 2018a). More precisely, Kurby and Zacks (2018a) showed that the segmentation of an event was related to the associated goal hierarchy (with for instance the coarse-grained 
segmentation being related to higher-level goals) and that this relation was reduced in older participants. In that study, however, participants segmented videos of other people performing actions (e.g., pitching a tent), so it remains unclear whether these findings extend to personally experienced events.

\section{Subjective experience of remembering}

The last aim of the current study was to shed some light on the impact of age on the subjective experience accompanying mental replay. We found that older adults assigned higher subjective ratings to their memories than young adults, in line with previous evidence that older adults often provide similar or higher subjective memory ratings than young adults (Comblain, D’Argembeau, \& Van Der Linden, 2005; De Brigard et al., 2016; Hashtroudi et al., 1990; Robin \& Moscovitch, 2017; St-Laurent et al., 2014). While in some of these studies, intact subjective ratings contrasted with impaired objective memory recall (Robin \& Moscovitch, 2017; St-Jacques et al., 2012; Folville et al., in press), older adults retrieved similar numbers of moments of prior experience as young adults in the current study. Interestingly, however, the number of recalled experience units predicted subjective vividness ratings in young but not in older adults, which suggests that older adults did not necessarily rely on the recall of experience units to assess the vividness of their memories. Nevertheless, in both age groups, the number of experience units significantly predicted the subjective sense of reliving the event and subjective memory for visual details, spatial information, and thoughts. It is possible that the kind of information vividness refers to is interpreted differently in older adults, so that they monitor retrieved details differently than young adults to make their vividness judgements (see Folville et al., in press and Mitchell \& Hill, 2019, for further discussion on this point). Finally, it is also worth noting that, in both 
age groups, subjective memory judgments were unrelated to the number of components retrieved within experience units, which replicates previous observations (Jeunehomme \& D'Argembeau, 2019). These findings suggest that, at least for complex real-life events, the subjective sense of re-experiencing the past depends on the quantity of moments that represent the unfolding of events rather than the amount of episodic details represented within these moments.

\section{Conclusion}

The present study shows that the temporal compression of daily life events in episodic memory is largely spared in aging. When mentally replaying the unfolding events, the density of recalled moments of prior experience did not differ between age groups and this density predicted memory compression rates to a similar extent in young and older adults. Furthermore, differences in compression rates as a function of the type of remembered events (actions vs. spatial displacements) were also comparable in the two age groups. On the other hand, some aspects of the subjective experience of remembering differed between age groups, with vividness ratings being less calibrated to objective recall in older than younger adults. Taken together, these results suggest that the mechanism of temporal compression of the flow of events in episodic memory is not affected by aging, but that the subjective vividness of memories become less tied to the objective amount of information that represents the unfolding of events. 


\section{References}

Aizpurua, A., \& Koutstaal, W. (2015). A matter of focus: Detailed memory in the intentional autobiographical recall of older and younger adults. Consciousness and Cognition, 33, 145-155. https://doi.org/10.1016/j.concog.2014.12.006

Allé, M. C., Manning, L., Potheegadoo, J., Coutelle, R., Danion, J. M., \& Berna, F. (2017). Wearable Cameras Are Useful Tools to Investigate and Remediate Autobiographical Memory Impairment: A Systematic PRISMA Review. Neuropsychology Review, 27(1), 81-99. https://doi.org/10.1007/s11065-016-9337-x

Arnold, A. E. G. F., Iaria, G., \& Ekstrom, A. D. (2016). Mental simulation of routes during navigation involves adaptive temporal compression. Cognition, 157, 14-23. https://doi.org/10.1016/j.cognition.2016.08.009.Mental

Baldassano, C., Chen, J., Zadbood, A., Pillow, J. W., Hasson, U., \& Norman, K. A. (2017). Discovering Event Structure in Continuous Narrative Perception and Memory. Neuron, 95(3), 709-721.e5. https://doi.org/10.1016/j.neuron.2017.06.041

Bastin, C., Diana, R. a, Simon, J., Collette, F., Yonelinas, A. P., \& Salmon, E. (2014). Associative memory in aging: The effect of unitization on source memory. Psychol Aging, 28(1), 275-283. https://doi.org/10.1037/a0031566.Associative

Bonasia, K., Blommesteyn, J., \& Moscovitch, M. (2016). Memory and navigation: Compression of space varies with route length and turns. Hippocampus, 26(1), 9-12. https://doi.org/10.1002/hipo.22539

Cabeza, R., Anderson, N. D., Locantore, J. K., \& McIntosh, A. R. (2002). Aging gracefully: Compensatory brain activity in high-performing older adults. Neurolmage, 17(3), 13941402. https://doi.org/10.1006/nimg.2002.1280

Cabeza, R., Nyberg, L., \& Park, D. C. (2016). Cognitive neuroscience of aging: Linking cognitive and cerebral aging. (O. U. Press, Ed.).

Chalfonte, B. L., \& Johnson, M. K. (1996). Feature memory and binding in young and older adults. Memory \& Cognition, 24(4), 403-416. https://doi.org/10.3758/BF03200930

Chen, J., Leong, Y. C., Honey, C. J., Yong, C. H., Norman, K. A., \& Hasson, U. (2017). Shared 
memories reveal shared structure in neural activity across individuals. Nature Neuroscience, 20(1), 115-125. https://doi.org/10.1038/nn.4450

Chow, T. E., \& Rissman, J. (2017). Neurocognitive mechanisms of real-world autobiographical memory retrieval: Insights from studies using wearable camera technology. Annals of the New York Academy of Sciences, 1396(1), 202-221. https://doi.org/10.1111/nyas.13353

Clewett, D., \& Davachi, L. (2017). The Ebb and Flow of Experience Determines the Temporal Structure of Memory. Current Opinion in Behvioural Science, 17, 186-193. https://doi.org/10.1037/h0076782

Comblain, C., D’Argembeau, A., \& Van Der Linden, M. (2005). Phenomenal characteristics of autobiographical memories for emotional and neutral events in older and younger adults. Experimental Aging Research, 31, 173-189.

Conway, M. A. (2009). Episodic memories. Neuropsychologia, 47, 2305-2313. https://doi.org/10.1016/j.neuropsychologia.2009.02.003

Davidson, P. S. R., \& Glisky, E. L. (2002). Neuropsychological correlates of recollection and familiarity in normal aging. Cognitive, Affective and Behavioral Neuroscience, 2(2), 174186. https://doi.org/10.3758/CABN.2.2.174

De Brigard, F., Giovanello, K. S., Stewart, G. W., Amber, W., Brien, M. M. O., Spreng, R. N., ... Stewart, G. W. (2016). Characterizing the subjective experience of episodic past, future , and counterfactual thinking in healthy younger and older adults. Quarterly Journal of Experimental Psychology, 69, 2358-2375.

https://doi.org/10.1080/17470218.2015.1115529

Deltour, J, J. (1993). Echelle de vocabulaire de Mill Hill de J. C. Raven. Adaptation française et normes comparées du Mill Hill et du Standard Progressive Matrice (PM 38): Manuel.

Duarte, A., Henson, R. N., \& Graham, K. S. (2008). The effects of aging on the neural correlates of subjective and objective recollection. Cerebral Cortex, 18(September), 2169-2180. https://doi.org/10.1093/cercor/bhm243

Duarte, A., Ranganath, C., Trujillo, C., \& Knight, R. T. (2006). Intact recollection memory in 
high-performing older adults: ERP and behavioral evidence. Journal of Cognitive Neuroscience, 18, 33-47. https://doi.org/10.1162/089892906775249988

Faber, M., \& Gennari, S. P. (2015). In search of lost time: Reconstructing the unfolding of events from memory. Cognition, 143, 193-202. https://doi.org/10.1016/j.cognition.2015.06.014

Faul, F., Erdfelder, E., Lang, A.-G., \& Buchner, A. (2007). G*Power 3: A flexible statistical power analysis program for the social, behavioral, and biomedical sciences. Behavior Research Methods, 39(2), 1202-1206. https://doi.org/10.1109/ISIT.2013.6620417

Field, A. P., \& Wilcox, R. R. (2017). Robust statistical methods: A primer for clinical psychology and experimental psychopathology researchers. Behaviour Research and Therapy, 98, 19-38. https://doi.org/10.1016/j.brat.2017.05.013

Folville, A., Bahri, M. A., Delhaye, E., Salmon, E., D’Argembeau, A., \& Bastin, C. (2019). Agerelated differences in the neural correlates of vivid remembering. Neuroimage. https://doi.org/https://doi.org/10.1016/j.neuroimage.2019.116336

Folville, A., D’Argembeau, A., \& Bastin, C. (in press). Deciphering the Relationship between Objective and Subjective Aspects of Recollection in Healthy Aging. Memory. https://doi.org/https://doi.org/10.1080/09658211.2020.1720741

Gaesser, B., Sacchetti, D, D., Addis, D. R., \& Schacter, D. L. (2011). Characterizing age-related changes in remembering the past and imagining the future. Psychology and Aging, 191(10 SUPPL), 80-84. https://doi.org/10.1037/a0021054.Characterizing

Gallo, D. A, Korthauer, L. E., Mcdonough, I. M., Teshale, S., \& Johnson, Elizabeth, L. (2011). Age-related positivity effects and autobiographical memory detail: evidence from a past/future source memory task. Memory, 19(6), 641-652. https://doi.org/10.1080/09658211.2011.595723.Age-Related

Hard, B. M., Recchia, G., \& Tversky, B. (2011). The shape of action. Journal of Experimental Psychology: General, 140(4), 586-604. https://doi.org/10.1037/a0024310

Hashtroudi, S., Johnson, M. K., \& Chrosniak, L. D. (1990). Aging and Qualitative Characteristics of Memories for Perceived and Imagined Complex Events. Psychology 
and Aging, 5, 119-126.

Jeunehomme, O., \& D’Argembeau, A. (in press). Event segmentation and the temporal compression of experience in episodic memory. Psychological Research, 0(0), 1-10. https://doi.org/10.1007/s00426-018-1047-y

Jeunehomme, O., \& D’Argembeau, A. (2019). The time to remember: temporal compression and duration judgments in memory for real-life events. Quarterly Journal of Experimental Psychology, 72(4), 930-942. https://doi.org/10.1177/1747021818773082

Jeunehomme, O., Folville, A., Stawarczyk, D., Van der Linden, M., \& D’Argembeau, A. (2018). Temporal compression in episodic memory for real-life events. Memory, 26(6), 759770. https://doi.org/10.1080/09658211.2017.1406120

Koen, J. D., \& Yonelinas, A. P. (2014). Recollection, not familiarity, decreases in healthy ageing: Converging evidence from four estimation methods. Memory (Hove, England), 8211(January 2015), 1-14. https://doi.org/10.1080/09658211.2014.985590

Koller, M. (2016). robustImm : An R Package for Robust Estimation of Linear Mixed-Effects Models. Journal of Statistical Software, 75(6). https://doi.org/10.18637/jss.v075.i06

Koo, T. K., \& Li, M. Y. (2016). A Guideline of Selecting and Reporting Intraclass Correlation Coefficients for Reliability Research. Journal of Chiropractic Medicine, 15(2), 155-163. https://doi.org/10.1016/j.jcm.2016.02.012

Kurby, C. A., \& Zacks, J. M. (2008). Segmentation in the perception and memory of events. Trends in Cognitive Sciences, 12(2), 72-79. https://doi.org/10.1016/j.tics.2007.11.004

Kurby, C. A., \& Zacks, J. M. (2011). Age differences in the perception of hierarchical structure in events. Memory and Cognition, 39(1), 75-91. https://doi.org/10.3758/s13421-0100027-2

Kurby, C. A., \& Zacks, J. M. (2018a). Age Differences in the Perception of Goal Structure in Everyday Activity. Psychology and Aging, 34(2), 187-201. https://doi.org/10.1037/pag0000321

Kurby, C. A., \& Zacks, J. M. (2018b). Preserved Neural Event Segmentation in Healthy Older Adults. Psychol Aging, 154(11), 2262-2265. 
https://doi.org/10.1016/j.pain.2013.06.005.Re-Thinking

Levine, B., Svoboda, E., Hay, J. F., Winocur, G., \& Moscovitch, M. (2002). Aging and autobiographical memory: Dissociating episodic from semantic retrieval. Psychology and Aging, 17(4), 677-689. https://doi.org/10.1037//0882-7974.17.4.677

Madore, K. P., Gaesser, B., \& Schacter, D. L. (2014). Constructive episodic simulation: dissociable effects of a specificity induction on remembering, imagining, and describing in young and older adults. Journal of Experimental Psychology. Learning, Memory, and Cognition, 40(3), 609-622. https://doi.org/10.1037/a0034885

Magliano, J., Kopp, K., Mcnerney, M. W., Radvansky, G. A., \& Zacks, J. M. (2012). Aging and perceived event structure as a function of modality. Aging, Neuropsychology, and Cognition, 5585(February). https://doi.org/10.1080/13825585.2011.633159

Mair, A., Poirier, M., \& Conway, M. A. (2017). Supporting older and younger adults' memory for recent everyday events: A prospective sampling study using SenseCam. Consciousness and Cognition, 49(February), 190-202. https://doi.org/10.1016/j.concog.2017.02.008

Mair, P., \& Wilcox, R. (2019). Robust statistical methods in R using the WRS2 package. Behavior Research Methods, (May). https://doi.org/10.3758/s13428-019-01246-w Mattis, S. (1976). Mental Status Examination for Organic Mental Syndrome in the Elderly Patient. In Geriatric Psychiatry.

McDonough, I. M., Cervantes, S. N., Gray, S. J., \& Gallo, D. a. (2014). Memory's aging echo: Age-related decline in neural reactivation of perceptual details during recollection. Neurolmage, 98, 346-358. https://doi.org/10.1016/j.neuroimage.2014.05.012

Michelmann, S., Staresina, B. P., Bowman, H., \& Hanslmayr, S. (2019). Speed of timecompressed forward replay flexibly changes in human episodic memory. Nature Human Behaviour, 3(2), 143-154. https://doi.org/10.1038/s41562-018-0491-4

Mitchell, K. J., \& Hill, E. M. (2019). The Impact of Focusing on Different Features During Encoding on Young and Older Adults' Source Memory. Open Psychology, 1(1), 106-118. https://doi.org/10.1515/psych-2018-0008 
Norman, K. A., \& Schacter, D. L. (1997). False recognition in younger and older adults :

Exploring the characteristics of illusory memories. Memory \& Cognition, 25(September), 838-848. https://doi.org/10.3758/BF03211328

Old, S. R., \& Naveh-Benjamin, M. (2008). Differential Effects of Age on Item and Associative Measures of Memory: A Meta-Analysis. Psychology and Aging, 23(1), 104-118. https://doi.org/10.1037/0882-7974.23.1.104

Pedraza, O., Lucas, J. A., Smith, G. E., Petersen, R. C., Graff-Radford, N. R., \& Ivnik, R. J. (2010). Robust and expanded norms for the dementia rating scale. Archives of Clinical Neuropsychology, 25(5), 347-358. https://doi.org/10.1093/arclin/acq030

R Core Team. (2013). R: A language and environment for statistical computing. R Foundation for Statistical Computing.

Radvansky, G. A., \& Curiel, J. M. (1998). Narrative comprehension and aging: The fate of completed goal information. Psychology and Aging, 13(1), 69-79. https://doi.org/10.1037/0882-7974.13.1.69

Radvansky, G. A., \& Dijkstra, K. (2007). Aging and situation model processing. Psychonomic Bulletin and Review, 14(6), 1027-1042. https://doi.org/10.3758/BF03193088

Radvansky, G. A., Krawietz, S. A., \& Tamplin, A. K. (2011). Walking through doorways causes forgetting: Further explorations. Quarterly Journal of Experimental Psychology, 64(8), 1632-1645. https://doi.org/10.1080/17470218.2011.571267

Radvansky, G. A., Pettijohn, K. A., \& Kim, J. (2015). Walking through doorways causes forgetting: Younger and older adults. Psychology and Aging, 30(2), 259-265. https://doi.org/10.1037/a0039259

Radvansky, G. A., \& Zacks, J. M. (2017). Event boundaries in memory and cognition. Current Opinion in Behvioural Science, 17, 133-140. https://doi.org/10.1016/j.cobeha.2017.08.006

Radvansky, G. A., Zwaan, R. A., Curiel, J. M., \& Copeland, D. E. (2001). Situation Models and Aging. Psychology and Aging, 16(1), 145-160.

Robin, J., \& Moscovitch, M. (2017). Familiar Real-World Spatial Cues Provide Memory 
Benefits in Older and Younger Adults. Psychology and Aging, 32, 210-219.

https://doi.org/10.1037/pag0000162

Silva, A. R., Pinho, M. S., Macedo, L., \& Moulin, C. J. A. (2018). A critical review of the effects of wearable cameras on memory. Neuropsychological Rehabilitation, 28(1), 117-141. https://doi.org/10.1080/09602011.2015.1128450

Spencer, W. D., \& Raz, N. (1995). Differential effects of aging on memory for content and context: a meta-analysis. Psychology and Aging, 10, 527.

St-Jacques, P., Rubin, D. C., \& Cabeza, R. (2012). Age-related effects on the neural correlates of autobiographical memory retrieval. Neurobiology of Aging, 33(7), 1298-1310. https://doi.org/10.1016/j.neuroimage.2010.01.051

St-Laurent, M., Abdi, H., Bondad, A., \& Buchsbaum, B. R. (2014). Memory Reactivation in Healthy Aging: Evidence of Stimulus-Specific Dedifferentiation. Journal of Neuroscience, 34(12), 4175-4186. https://doi.org/10.1523/JNEUROSCI.3054-13.2014

St-laurent, M., Abdi, H., Burianova, H., \& Grady, C. L. (2011). Influence of Aging on the Neural Correlates of Autobiographical, Episodic and Semantic Memory Retrieval. Journal of Cognitive Neuroscience, 23(12), 4150-4163. https://doi.org/10.1162/jocn

Stine-Morrow, E. A. L., Soederberg Miller, L. M., \& Leno, R. (2001). Patterns of on-line resource allocation to narrative text by younger and older readers. Aging, Neuropsychology, and Cognition, 8(1), 36-53. https://doi.org/10.1076/anec.8.1.36.848

Wang, Y., \& Gennari, S. P. (2019). How language and event recall can shape memory for time. Cognitive Psychology, 108(October 2018), 1-21.

https://doi.org/10.1016/j.cogpsych.2018.10.003

Wilcox, R. R. (2012). Introduction to robust estimation and hypothesis testing. Waltham: Academic Press.

Zacks, J. M., Speer, N. K., Swallow, K. M., Braver, T. S., \& Reynolds, J. R. (2007). Event Perception: A Mind/Brain Perspective. Psychological Bulletin, 133(2), 273-293. https://doi.org/10.1037/0033-2909.133.2.273.Event

Zacks, J. M., Speer, N. K., Vettel, J. M., \& Jacoby, L. L. (2006). Event understanding and 
memory in healthy aging and dementia of the alzheimer type. Psychology and Aging, 21(3), 466-482. https://doi.org/10.1037/0882-7974.21.3.466 


\section{Figures captions}

Figure 1. Overview of experienced events. Participants had to go to different locations to perform specific actions. On the map, actions are represented by circles and spatial displacements are symbolised by lines. An example of a picture taken by the wearable camera is presented for each event.

Figure 2. Schematic representation of a trial of the memory task. Each trial began with the presentation of two pictures and one title describing the corresponding event. Participants first had to mentally relive the event as precisely as they could and the duration of their mental replay was measured. Then, participants were presented with six rating scales assessing several subjective memory dimensions. Finally, participants verbally described everything they remembered during their mental replay and their verbal reports were recorded.

Figure 3. Rates of temporal compression as a function of age and type of events. Bars represent the $20 \%$ trimmed means and error-bars represent $95 \%$ robust confidence intervals.

Figure 4. Density of recalled experience units as a function of age and type of events. Bars represent the $20 \%$ trimmed means and error-bars represent $95 \%$ robust confidence intervals. 
Figure 1.

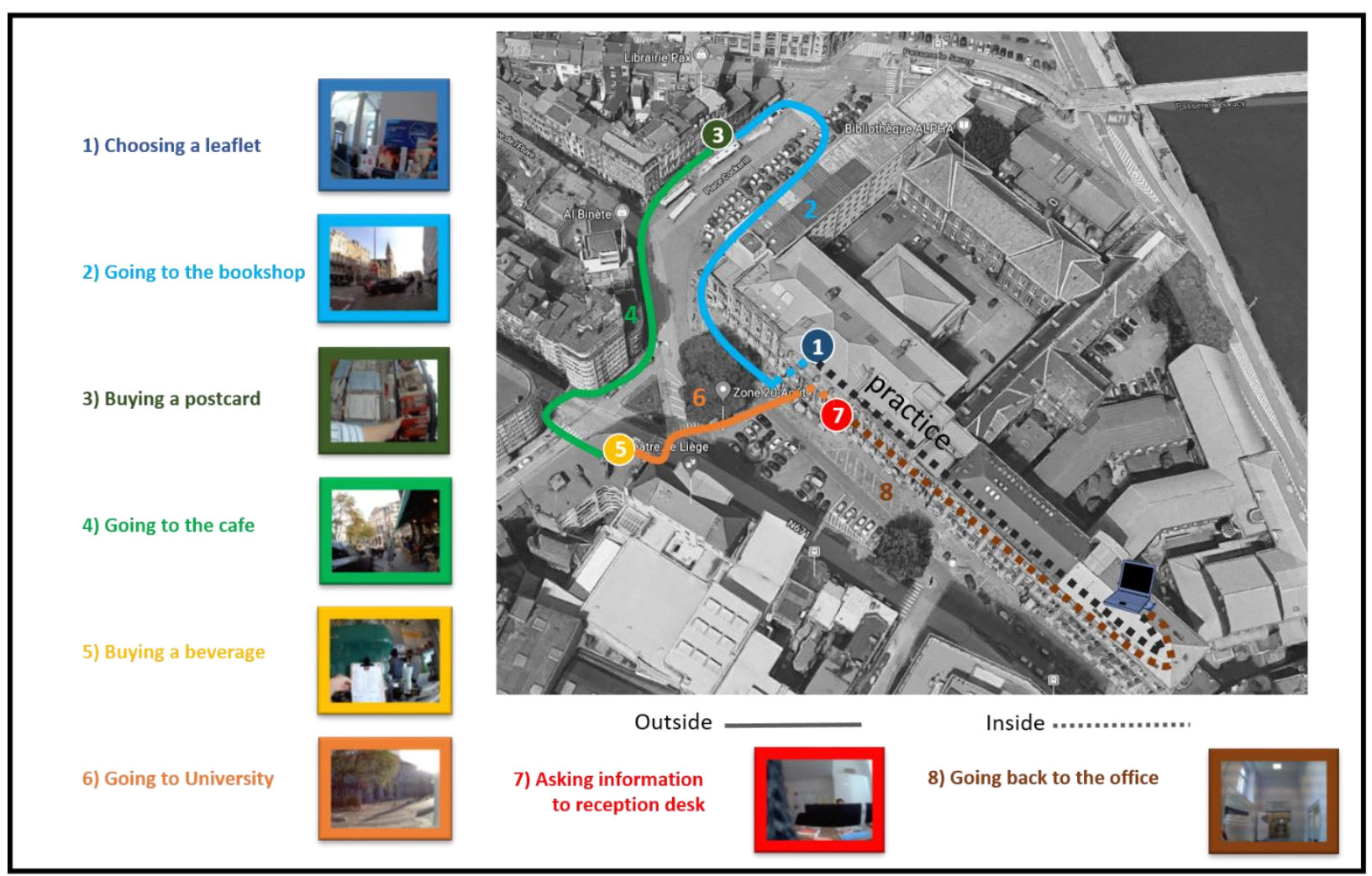


Figure 2.

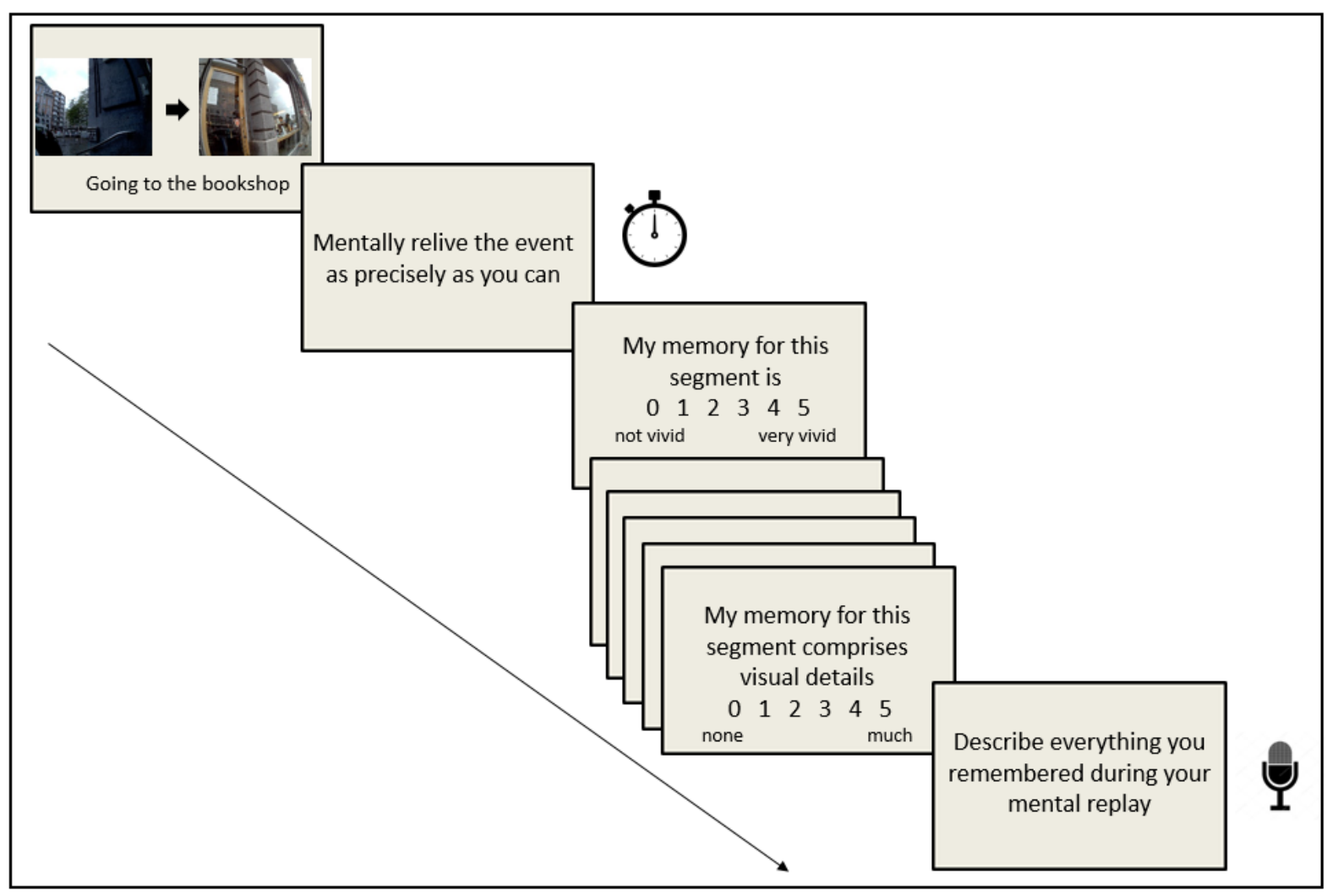


Figure 3.

11

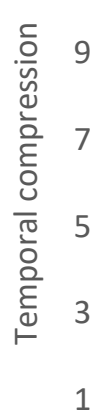

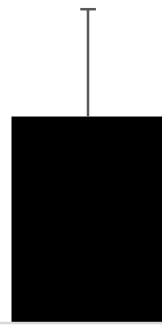

Goal-directed actions

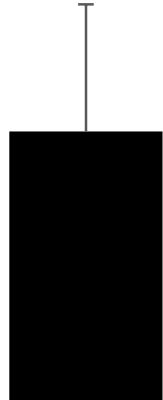

Spatial displacements

-Young Older 
Figure 4.

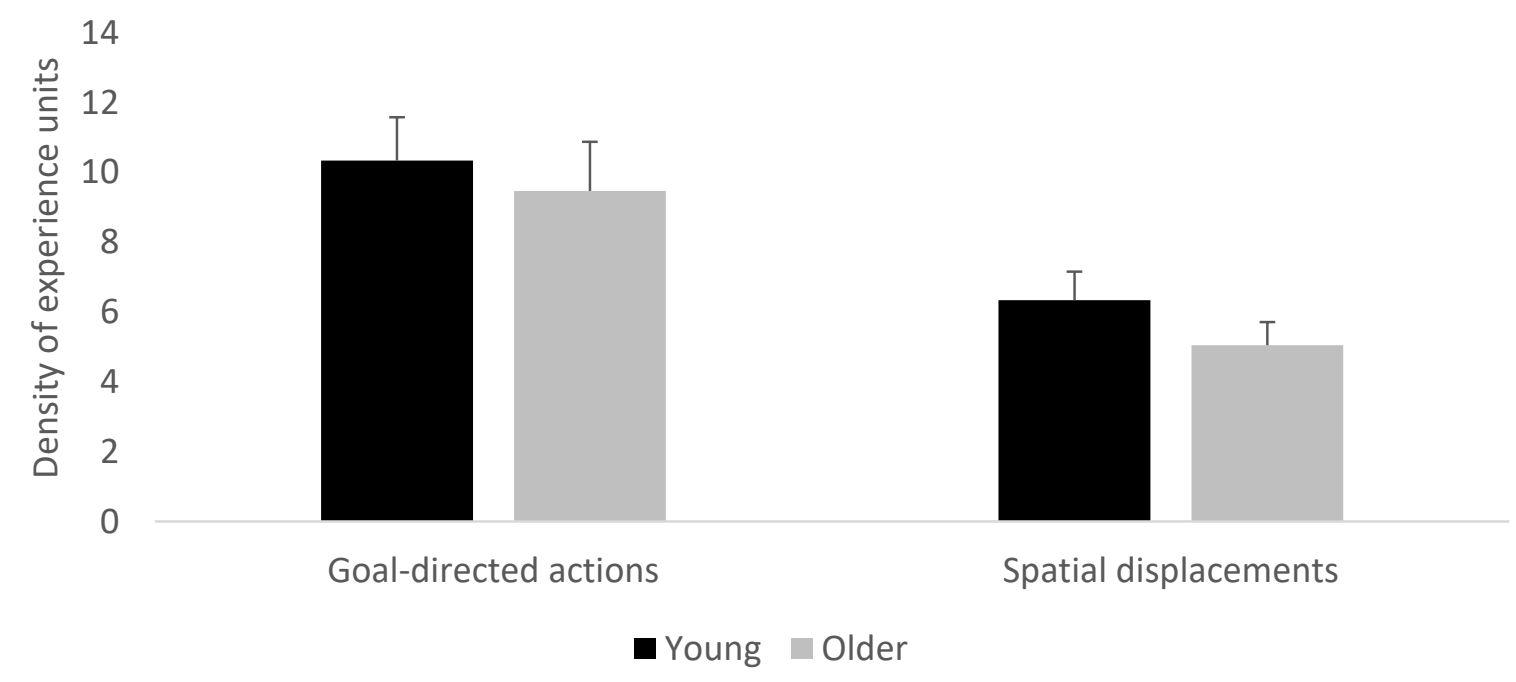


Table 1. Descriptions and examples of the different categories of components constituting experience units.

\section{Component categories}

Person

Object

Thought

Action with interaction

Spatial movement

Perceptual detail

\section{Descriptions and examples}

Description of one or more person(s) with no description of interacting with this/these person(s).

"I saw a woman"

"There was a group of students"

Description of an object or aspect of the external environment, with no description of interacting with this object (if an interaction was described, the component was classified as "action with interaction").

"I saw a car"

"The sun was shining"

Description of a thought, mental state or judgement.

"I was lost in my thoughts"

"She seemed upset"

Description of an action performed by the participant involving a direct interaction with a person or an object.

"I opened the door" "she gave me the coffee"

Description of a movement of the body in the environment.

"I turned right"

"I walked to the office"

Description of a sensory detail (i.e. colour, texture, shape...) about a person or an object, or of an internal sensation.

"She wore black sunglasses"

"I had a stomach ache" 
Spatial detail

Comment
Description of a detail replacing a person or an object in the spatial context.

"A man walked in front of me"

"The leaflets were on my left"

Explanation or clarification that does not in itself describes the past experience.

"I never put sugar in my coffee"

Note: In each example that is provided, the corresponding component is highlighted in bold 
Table 2. Multilevel regression analyses assessing the effects of age and recalled experience units on the subjective experience of remembering.

\begin{tabular}{|c|c|c|c|c|c|c|c|c|c|c|c|c|}
\hline \multirow[t]{2}{*}{$\begin{array}{l}\text { Outcome } \\
\text { variable }\end{array}$} & \multicolumn{3}{|c|}{ Number of experience units } & \multicolumn{3}{|c|}{ Age-group } & \multicolumn{3}{|c|}{$\begin{array}{l}\text { Number of experience units } \\
\text { x Age-group interaction }\end{array}$} & \multicolumn{3}{|c|}{$\begin{array}{l}\text { Components within } \\
\text { experience units }\end{array}$} \\
\hline & $\beta$ & $S E$ & $t$ & $\beta$ & $S E$ & $t$ & $\beta$ & $S E$ & $t$ & $\beta$ & $S E$ & $t$ \\
\hline Vividness & 0.161 & 0.046 & $3.51^{*}$ & 0.705 & 0.147 & $4.79^{*}$ & -0.154 & 0.073 & $-2.09 *$ & -0.020 & 0.035 & -0.57 \\
\hline Reliving & 0.123 & 0.030 & $4.03^{*}$ & 0.774 & 0.169 & $4.58^{*}$ & -0.068 & 0.049 & -1.36 & -0.040 & 0.023 & -1.71 \\
\hline
\end{tabular}

Note. Significant predictors $(p<.05)$ are indicated by an asterisk. All components within experience units except comments were entered in the model. 
The impact of age on the temporal compression of daily life events in episodic memory

Adrien Folville, Olivier Jeunehomme, Christine Bastin \& Arnaud D’Argembeau

University of Liège

Supplementary material 


\section{Event duration}

We conducted a robust 2 (age) $\times 2$ (event type) mixed ANOVA on event durations. It revealed neither a main effect of event type, $F_{t}(1,41.58)=1.19, p=.28, \xi=0.18$, nor a main effect of age, $F_{t}(1,41.12)=0.84, p=.36, \xi=0.19$, but the age $x$ event type interaction was significant, $F_{t}(1,41.58)=6.05, p=.02$ (see Table S1 for mean event durations according to event type and age group). Follow-up comparisons using robust t-test revealed that event duration did not differ between young and older adults for actions, $\mathrm{Mdiff}_{\mathbf{f}}=36.72,95 \% \mathrm{Cl}[-$ 56.34, 129.79], $Y_{t}(41.91)=0.79, p=.43, \xi=0.14$, but was significantly higher in older than young adults for spatial displacements, $M_{\text {diff }}=84.36,95 \% \mathrm{Cl}[42.05,126.67], \mathrm{Y}_{\mathrm{t}}(41.91)=$ $4.02, p<.001, \xi=0.63$. These results indicate that young and older participants took the same time to perform the requested actions at a given location but that older participants took more time to go from one place to another, probably because they walked more slowly than young adults.

Table S1. 20\% trimmed means and $95 \% \mathrm{Cl}$ of event duration (in seconds) as a function of age and type of events.

Event type

Age group $\quad$ Action $\quad$ Spatial displacement

\begin{tabular}{lcc}
\hline Young & $449.72[387.81,516.63]$ & $416.09[387.72,449.72]$ \\
Older & $413[355.82,478-82]$ & $500.45[470.09,539.90]$
\end{tabular}




\section{Relationship between event duration and temporal compression}

Next, we examined whether rates of temporal compression vary with event duration. We conducted a robust multilevel regression analysis with temporal compression rate as dependent variable and event duration as first-level predictor. This analysis revealed that temporal compression rates increased with event duration, $\beta=250, S E=0.011, t=22.39, p$ $<.001$ (see Figure S1). Because spatial displacements were longer for older than young adults, we further examined the effect of age on temporal compression while controlling for event duration. We conducted a robust multilevel regression analysis with temporal compression rate as dependent variable and with event duration and age group as first- and second-level predictors, respectively. A cross-level interaction (duration $\mathrm{x}$ age) was also modelled to examine potential age differences in the relationship between event duration and temporal compression. This analysis was conducted for spatial displacements only because no age difference in event duration was found for actions (see above). ${ }^{4}$ This analysis revealed that event duration predicted temporal compression rates, $\beta=0.220, S E=$ $0.027, t=8.20, p<.001$, but the effect of age, $\beta=-0.003, S E=0.080, t=-0.049$, and the event duration by age interaction, $\beta=0.036, S E=0.031, t=1.14$, were not significant. The absence of interaction indicates that event duration influenced temporal compression in a similar way for young and older participants.

\footnotetext{
${ }^{4}$ It should also be noted that actions and spatial displacements had a comparable duration in young adults, $M_{\text {diff }}=33.63,95 \% \mathrm{Cl}[-35.02,102.29], \mathrm{Yt}(21)=1.02, p=.32, \xi=0.18$, such that the observed difference in temporal compression rates between the two types of events cannot be attributed to a difference in event duration.
} 
Figure S1. Relationship between temporal compression and event duration as modelled in the multilevel model.

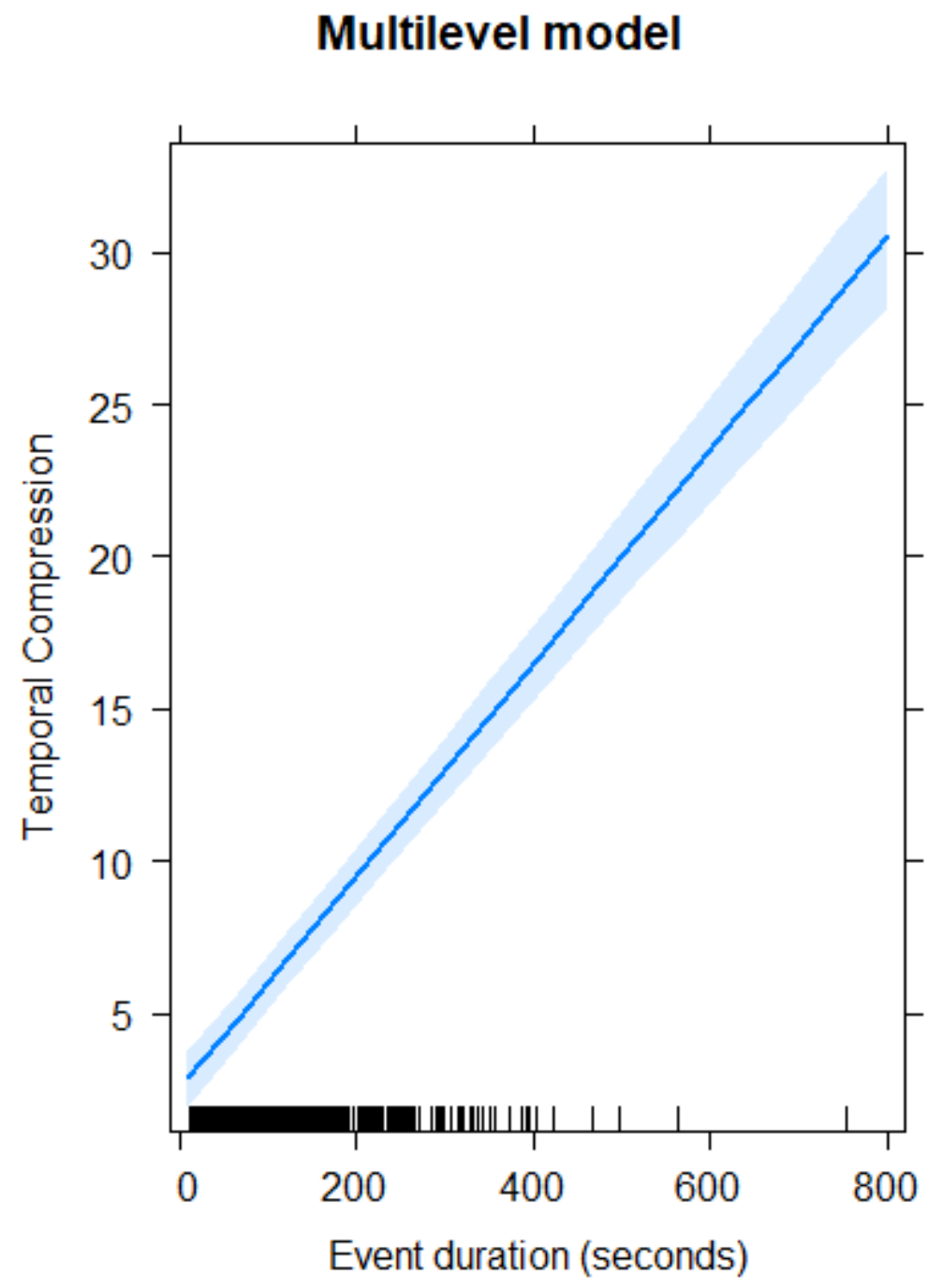

\section{Components of recalled experience units}

Robust 2 (age) $\times 2$ (event type) mixed ANOVAs were conducted for each component category: person, object, thought, action with interaction, spatial movement, perceptual detail, spatial detail, and comment (see Figure S2 and Table S2). The main effect of age was significant for the comment category (indicating that older adults made more comments than young adults), but was not significant for the remaining components. There was a main 
effect of event type for the object, action with interaction, spatial movement, and comment categories; for object and spatial movement categories, the number of components was higher for spatial displacements than actions, whereas for action with interaction and comment categories, the number of components was higher for actions than spatial displacements. There was no age by event type interaction in any of the ANOVAs.

Figure S2. Mean number of components within experience units as a function of types of events and age groups. Bars represent the $20 \%$ trimmed means and error-bars represent $95 \%$ robust confidence intervals.

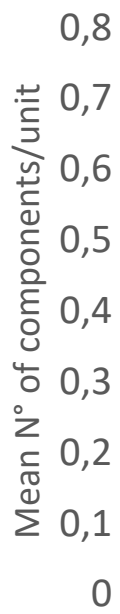

0

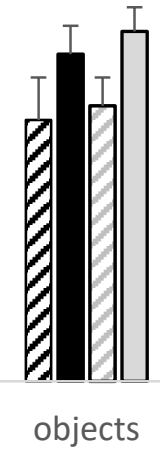

objects

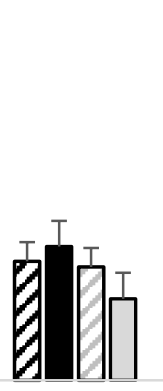

people thoughts

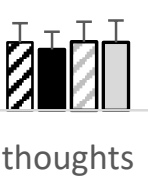

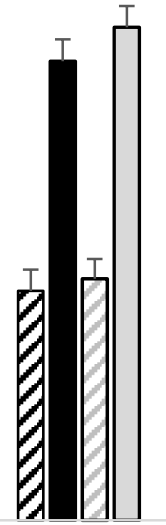

movements

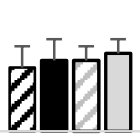

spatial details

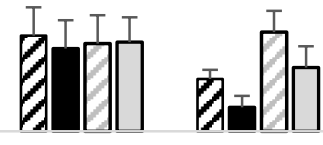

perceptive comments details

Component categories 
Table S2. Results of the robust 2 (age) $\times 2$ (event type) mixed ANOVAs.

\begin{tabular}{|c|c|c|c|c|c|c|c|c|}
\hline \multirow[t]{2}{*}{$\begin{array}{l}\text { Component } \\
\text { category }\end{array}$} & \multicolumn{3}{|c|}{ Age } & \multicolumn{3}{|c|}{ Event-type } & \multicolumn{2}{|c|}{ Age $x$ Event-type interaction } \\
\hline & $F_{t}$ & $p$ & $\xi$ & $F_{t}$ & $p$ & $\xi$ & $F_{t}$ & $p$ \\
\hline Person & $(1,38.06)=2.94$ & .095 & 0.31 & $(1,40.95)=0.97$ & .331 & 0.11 & $(1,40.95)=3.72$ & .065 \\
\hline Object & $(1,31.60)=1.07$ & .308 & 0.22 & $(1,35.92)=44.83$ & $<.001$ & 0.59 & $(1,35.92)=0.19$ & .663 \\
\hline Thought & $(1,41.56)=0.07$ & .797 & 0.06 & $(1,38.27)=0.21$ & .646 & 0.03 & $(1,38.27)=1.95$ & .170 \\
\hline $\begin{array}{l}\text { Action with } \\
\text { interaction }\end{array}$ & $(1,41.94)=0.15$ & .704 & 0.10 & $(1,40.87)=393.85$ & .001 & 0.98 & $(1,40.87)=2.36$ & .131 \\
\hline $\begin{array}{c}\text { Spatial } \\
\text { movement }\end{array}$ & $(1,38.79)=3.63$ & .064 & 0.31 & $(1,41.96)=979.95$ & .001 & 0.99 & $(1,41.96)=2.09$ & .150 \\
\hline $\begin{array}{c}\text { Perceptive } \\
\text { detail }\end{array}$ & $(1,41.99)=0.01$ & .979 & 0.01 & $(1,41.93)=0.37$ & .543 & 0.06 & $(1,41.93)=0.53$ & .469 \\
\hline Spatial detail & $(1,39.67)=0.55$ & .459 & 0.14 & $(1,41.99)=0.71$ & .403 & 0.10 & $(1,41.99)=0.07$ & .785 \\
\hline Comment & $(1,31.02)=23.70$ & $<.001$ & 0.73 & $(1,37.67)=25.13$ & $<.001$ & 0.42 & $(1,37.67)=0.22$ & .639 \\
\hline
\end{tabular}




\section{Subjective remembering}

We examined the impact of age on subjective memory judgements and investigated whether these judgements were predicted by recalled experience units. For each subjective judgment, we conducted a robust multilevel regression analysis with the ratings of interest as outcome variable and with the number of recalled experience units and age group as first- and second-level predictors; the number of recalled units by age group cross-level interaction was also entered as predictor in the model to examine potential age differences in the relationship between the number of retrieved experience units and the associated subjective memory experience (see Table S3). Finally, the number of components recalled within experience units corresponding to each subjective memory scale (e.g., for the visual detail scale, all categories of recalled components that comprised visual details were summed: person, object, action with interaction, spatial movement, perceptual detail and spatial detail) was also entered in the model to examine their potential contribution to the subjective experience of remembering.

For all dimensions, the effects of the number of experience units and age group were significant (except the effect of number of experience units for order judgments; see Table S3). These effects indicated that ratings increased with the number of recalled experience units and that older adults provided higher ratings than young adults. The cross-level interaction was not significant, meaning that the number of recalled experience units predicted subjective experience to a similar extent in young and older adults. The number of components within experience units did not significantly predict subjective memory judgments. 
Table S3. Multilevel regression analyses assessing the effects of age and recalled experience units on the subjective experience of remembering.

\begin{tabular}{|c|c|c|c|c|c|c|c|c|c|c|c|c|}
\hline \multirow[t]{2}{*}{$\begin{array}{l}\text { Outcome } \\
\text { variable }\end{array}$} & \multicolumn{3}{|c|}{ Number of experience units } & \multicolumn{3}{|c|}{ Age-group } & \multicolumn{3}{|c|}{$\begin{array}{l}\text { Number of experience units } \\
\text { x Age-group interaction }\end{array}$} & \multicolumn{3}{|c|}{$\begin{array}{l}\text { Components within } \\
\text { experience units }\end{array}$} \\
\hline & $\beta$ & $S E$ & $t$ & $\beta$ & $S E$ & $t$ & $\beta$ & $S E$ & $t$ & $\beta$ & $S E$ & $t$ \\
\hline $\begin{array}{l}\text { Visual } \\
\text { details }\end{array}$ & 0.201 & 0.047 & $4.31^{*}$ & 0.723 & 0.158 & $4.57^{*}$ & -0.053 & 0.075 & -0.71 & 0.041 & 0.036 & 1.14 \\
\hline $\begin{array}{c}\text { Spatial } \\
\text { information }\end{array}$ & 0.199 & 0.049 & $4.03^{*}$ & 0.603 & 0.140 & $4.30 *$ & -0.116 & 0.040 & -1.45 & 0.011 & 0.036 & 0.29 \\
\hline Thought & 0.118 & 0.042 & $2.78^{*}$ & 0.505 & 0.150 & $3.35^{*}$ & -0.047 & 0.068 & -0.68 & 0.046 & 0.033 & 1.42 \\
\hline Order & -0.001 & 0.035 & -0.01 & 0.514 & 0.175 & $2.93^{*}$ & -0.001 & 0.056 & -0.01 & -0.030 & 0.026 & -1.14 \\
\hline
\end{tabular}

Note. Significant predictors $(p<.05)$ are indicated by an asterisk. For the thought variable, all components except comments within experience units were entered in the model. For the visual details variable analysis, all components except thoughts and comments were entered in the model. For the spatial information and thought variable analyses, Spatial and Thought components were entered in the model, respectively. 
